\title{
Detonation Velocity Measurement of a Hydrogen Peroxide Solvate of CL-20
}

\author{
Vasant S. Vuppuluri, ${ }^{[\mathrm{a}]}$ Jonathan C. Bennion, ${ }^{[\mathrm{b}, \mathrm{cc}}{ }^{\mathrm{Ren}}$ A. Wiscons, ${ }^{[\mathrm{b}]}$ I. Emre Gunduz, ${ }^{[\mathrm{d}]}$ Adam J. Matzger, ${ }^{[\mathrm{b}]}$ and \\ Steven F. Son ${ }^{[a]}$
}

\begin{abstract}
Synthesis and development of new energetic molecules is a resource-intensive process, yielding materials with relatively unpredictable performance properties. Cocrystallization and crystalline solvate formation have been explored as possible routes towards developing new energetic materials that reduce the initial investment required for discovery and performance uncertainty because existing energetic molecules with known properties serve as the constituents. The formation of a hydrogen peroxide (HP) solvate of CL-20 was previously reported and has a density comparable to that of $\varepsilon-C L-20$, the densest and most stable polymorph of CL-20. CL-20/HP produces a second crystalline form, which was unexpected given the high density of
\end{abstract}

Keywords: $\mathrm{CL}-20 \cdot$ solvate $\cdot$ detonation the original $\mathrm{CL}-20 / \mathrm{HP}$ solvate. Both forms were predicted to have improved detonation performance relative to that of $\varepsilon-C L-20$. In this work, the detonation velocity of a solvate of $\mathrm{CL}-20 / \mathrm{HP}$ is measured and compared to that of CL-20. Using the measured enthalpy of formation, the solvate was predicted to detonate $80 \mathrm{~m} \mathrm{~s}^{-1}$ faster at a powder density of $1.4 \mathrm{~g} \mathrm{~cm}^{-3}$; however, experimentally, the solvate detonates $300 \mathrm{~m} \mathrm{~s}^{-1}$ faster than CL-20. Thermochemical predictions are also used to show that the solvate detonates $100 \mathrm{~m} \mathrm{~s}^{-1}$ faster than $\varepsilon-C L-20$ at the theoretical maximum density, making it the first energetic cocrystal or solvate of $\varepsilon-C L-20$ predicted to detonate faster than CL-20 at full density.

\section{Introduction}

Successful development of novel energetic materials is challenging due to the difficulty associated with safely synthesizing viable molecules and extensive chemical and materials characterization required before qualifying for application. Cocrystallization is an attractive alternative path towards the development of energetic materials. Cocrystallization is a process by which two compounds are combined to yield a unique crystal structure containing both molecules from both compounds in a stoichiometric ratio. The cocrystal structure can yield unique detonation characteristics relative to the parent energetic compound(s) due to new intermolecular interactions formed and molecular conformations present in the structure. In particular, 2,4,6,8,10,12-hexanitro-2,4,6,8,10,12-hexaazoisowurtzitane $(\mathrm{CL}-20)$ has been used as a coformer in energetic cocrystals. A review of relevant work relating to energetic cocrystals of CL-20 is presented in Vuppuluri et al. [1].

Crystalline hydrates and solvates of energetic materials form in a process similar to cocrystallization but are distinct because one or more coformer(s) are liquid when pure at standard temperature and pressure [2]. In $\alpha-C L-20$, for example, water molecules can occupy intermolecular sites to produce either a $1 / 2$ or a $1 / 4$ hydrate (half of which are filled with water molecules). Both hydrate forms have lower crystal densities than $\varepsilon-C L-20$, which is the densest $\left(2.044 \mathrm{~g} \mathrm{~cm}^{-3}\right)$ and the least sensitive polymorph of $\mathrm{CL}-20$
[3]. Energetic hydrates often show poor density, which is undesirable as this reduces detonation performance. Similarly, crystalline solvates generally yield low-density forms in addition to reducing the oxygen balance of the material by introducing carbon-rich constituents that are not fully oxidized upon decomposition. For example, octahydro1,3,5,7-tetranitro-1,3,5,7-tetrazocine (HMX) is known to form a number of solvates, including a $\mathrm{N}, \mathrm{N}$-dimethylformamide (DMF) solvate [4]. Bennion et al. reported the formation of two hydrogen peroxide (HP) solvates of CL-20 [5]. Both sol-

[a] V. S. Vuppuluri, S. F. Son

Purdue University

Department of Mechanical Engineering

585 Purdue Mall,

West Lafayette, IN 47907, USA

[b] J. C. Bennion, R. A. Wiscons, A. J. Matzger

University of Michigan

Department of Chemistry

930N. University Ave.

Ann Arbor, MI, 48109, USA

[c] J. C. Bennion

Energetic Materials Science Branch

Army Research Laboratory

Aberdeen Proving Ground, MD, 21005, USA

[d] I. E. Gunduz

Naval Postgraduate School

Mechanical and Aerospace Engineering Department

Monterey, CA, 93943, USA 
vates form in a 2:1 molar ratio of CL-20 to HP, respectively, although one form crystallizes in an orthorhombic crystal system and the other in a monoclinic crystal system [5]. The densities of the orthorhombic and monoclinic solvates were measured using single crystal X-ray diffraction (SCXRD) to be $2.033 \mathrm{~g} \mathrm{~cm}^{-3}$ and $1.966 \mathrm{~g} \mathrm{~cm}^{-3}$, respectively [5]. In addition, the decomposition temperatures of both CL-20/HP solvates were found to be comparable to that of $\alpha-C L-20$ [5]. In the Supporting Information of Bennion et al., it was deteremined using Raman spectroscopy that the endotherms observed for both CL-20/HP solvate forms corresponds to the release of hydrogen peroxide [5]. Bennion et al. predicted, that at its theoretical maximum density (TMD), the orthorhombic solvate (treated as a formulation of the components) would detonate approximately $160 \mathrm{~m} \mathrm{~s}^{-1}$ faster than $\varepsilon-C L-20$ using the thermochemical code CHEETAH 7.0. Previous research groups have shown that intermolecular interactions between coformers and conformational differences in molecular structure can modify detonation performance relative to a physical mixture of the neat starting components in small scale testing. For example, the energetic cocrystals of CL-20 reported thus far exhibit lower impact sensitivity compared to that of neat CL-20, although they were still measured to have relatively high densities [6-8]. That said, the effect of coformer interactions on largescale performance remains an open question, and particularly its effect on detonation velocity.

To evaluate performance of explosives at realistic scales, detonation experiments typically require several hundreds to several thousands of grams of material. This amount of material often exceeds the supply in the developmental stage. Furthermore, experiments at this scale are costly, necessitating preliminary small-scale detonation velocity measurements.

Microwave interferometry (MI) is a well-established technique for measuring detonation velocity on less than two grams of total material, with its use going as far back as the 1950s [9]. The principles relating MI measurements and detonation velocity are presented in Vuppuluri et al. [1]. Using MI, Vuppuluri et al. demonstrated that it was possible to obtain well-resolved measurements of detonation velocity with as little as $1.5 \mathrm{~g}$ of MDNT/CL-20 cocrystal. Further, it was shown by this method that the cocrystal detonates over $500 \mathrm{~m} \mathrm{~s}^{-1}$ faster than the physical mixture of the two energetic components. Using the enthalpy of formation measured by bomb calorimetry, CHEETAH predicted that the cocrystal would detonate approximately $230 \mathrm{~m} \mathrm{~s}^{-1}$ faster than the physical mix.

The objective of this work is to quantify the effect of solvate formation between $\mathrm{CL}-20$ and hydrogen peroxide on detonation velocity. To accomplish this, a method utilizing MI similar to that described above was employed to measure detonation velocity of the orthorhombic solvate of CL20/HP reported previously.

\section{Experimental Methods}

\subsection{Sample Preparation and Detonation Experiment}

CL-20 was used as received by Armament Research, Development, and Engineering Command (ARDEC) at Picatinny Arsenal. The orthorhombic CL-20/HP form was prepared by dissolving $\mathrm{CL}-20$ in dry acetonitrile and adding concentrated HP (>95\%) dropwise, precipitating the orthorhombic solvate as described in the Supporting Information section of Bennion et al. [5]. The preparation method for detonation test samples is identical to the method described in Vuppuluri et al. [1].

\subsection{Bomb Calorimetry}

Bomb calorimetry experiments were performed using a Parr 1281 Calorimeter with a 1108 C Oxygen Bomb. For each test, the bomb was filled with oxygen to a pressure of about $3 \mathrm{MPa}$ (30 atm). The nominal pellet mass was $0.3 \mathrm{~g}$. The calorimeter was calibrated using a benzoic acid standard in order to determine the heating value of the calorimeter. Using the gross heat of combustion and temperature from the calorimetry measurement, the enthalpy of combustion in $\mathrm{kJ} \mathrm{mol}^{-1}(\Delta H c)$ is calculated as:

$\Delta H_{c}=-\left(M u\left(4.186 \times 10^{-3}\right)+\Delta n R T\right)$,

where $M$ is the molecular weight, $\mathrm{u}$ is the gross heat of combustion in calg $^{-1}$ measured by the bomb calorimeter, $\Delta n$ is the net moles of gas produced, $R$ is the universal gas constant in $\mathrm{J} \mathrm{kg}^{-1} \mathrm{~mol}$ and $T$ is temperature.

For a CHNO explosive with the formula $\mathrm{C}_{x} \mathrm{H}_{y} \mathrm{~N}_{z} \mathrm{O}_{w}$ undergoing complete combustion, the net moles of gas produced $(\Delta n)$ is:

$\Delta n=-\frac{y}{4}+\frac{z}{2}+\frac{w}{2}$.

The enthalpy of formation $\Delta H_{f}^{\circ}$ is computed as:

$\Delta H_{f}^{\circ}=\frac{y}{2} \Delta H_{f, \mathrm{H}_{2} \mathrm{O}(l)}^{\circ}+x \Delta H_{f, \mathrm{CO}_{2}}^{\circ}-\Delta H_{c}$.

\subsection{Detonation Experiment}

A diagram of the small-scale detonation experiment is shown in Figure 1. One end of a $6.35 \mathrm{~mm}$ polytetrafluoroethylene (PTFE) waveguide rod is placed in contact with the sample material. The other end is inserted into a WR-28 waveguide on the $35 \mathrm{GHz}$ microwave interferometer. The charge is initiated with a Teledyne RISI RP-502 exploding bridge-wire (EBW) detonator. The fiber optic cable is connected to a Thorlabs DET10 A photodiode to detect light 


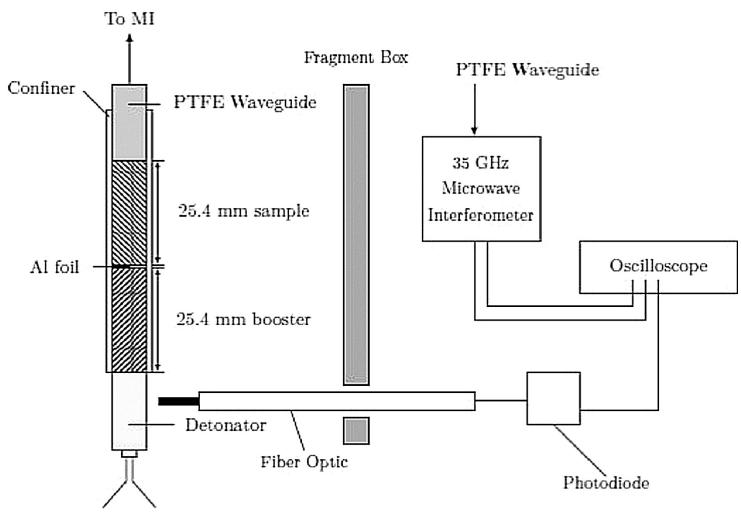

Figure 1. Diagram of detonation experiment [1] (used with permission).

from the breakout of the detonator. The photodiode triggers a Tektronix DPO4034 oscilloscope which records data from the in-phase and quadrature channels of the microwave interferometer. Further details of the detonation experiment are given in Vuppuluri et al. [1].

\subsection{Analysis Methods}

A sample interferometer waveform for the CL-20/HP solvate is shown in Figure 2. The in-phase and quadrature signals are denoted by $\mathrm{CH} 1$ and $\mathrm{CH} 2$ in Figure 2, respectively. For a sinusoidal waveform with exponentially varying amplitudes, a curve fit of the form:

$a e^{-b t} \sin (2 \pi f t+\Phi)+c$

is applied, where $a, b, f, \phi$ and $c$ are fitting constants. The

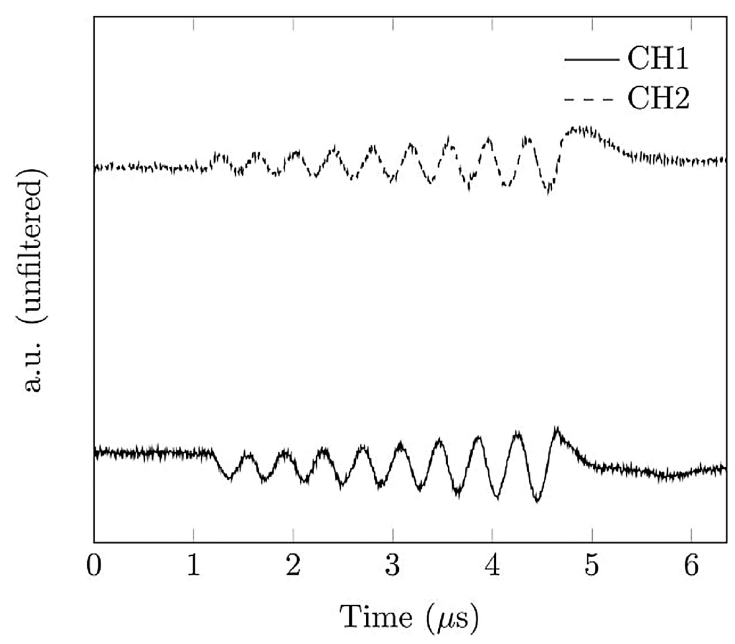

Figure 2. Typical unfiltered data from microwave interferometer. The solid line labeled $\mathrm{CH} 1$ is the in-phase signal and the dashed line labeled $\mathrm{CH} 2$ is the quadrature signal. value of $b$ is taken to be the average ratio of the amplitudes of successive peaks. The value of $f$ is taken to be the average time between successive peaks and $c$ is taken to be the average value of the waveform. The Levenberg-Marquardt algorithm is then used to determine the values of $a$ and $\phi$. The resulting curve-fit is used to estimate the start and end times of the detonation event.

After extracting the detonation event waveform from the full signal, a second-order Butterworth low-pass filter is applied. The filtered waveforms are then normalized by dividing the waveform by the maximum value and translating the waveform so that the amplitudes lie between $[-1,1]$. With an ideal quadrature detector system, the gain in the in-phase and quadrature signals would both be unity. However, normalization with a single normalization parameter fails to correct for the effect of unequal channel gain, which is one of the non-idealities present in real quadrature interferometry systems [10].

To mitigate the effect of unequal signal gain, a Hilbert transform is first applied to the two normalized signals. The Hilbert transform is a linear operator that generates an orthogonal complement to a given signal [11]. The original signal and its harmonic conjugate (orthogonal to the original signal) form the real and imaginary parts of the transformed signal such that the absolute value of the transformed signal gives the envelope function of the original signal. Each point in the normalized waveform is re-normalized to the envelope function.

Even after normalization, the Lissajous curves for the two channels still form a distorted ellipse due to other key non-idealities present in quadrature interferometry systems such as channel nonorthogonality and zero offset [10]. The cumulative effect of the nonidealities present in a quadrature detector system is modeled as stretching, rotation, and translation of a unit circle, yielding a distorted ellipse. The equation of this ellipse is

$$
\left[\begin{array}{l}
V_{1} \\
V_{2}
\end{array}\right]=\mathbf{Q}(\alpha)\left[\begin{array}{ll}
a & 0 \\
0 & b
\end{array}\right]\left[\begin{array}{l}
V_{1 c} \\
V_{2 c}
\end{array}\right]+\left[\begin{array}{l}
z_{x} \\
z_{y}
\end{array}\right],
$$

where $\mathbf{Q}(\alpha)$ represents the rotation matrix as a function of angle $\alpha, a$ and $b$ are respectively the major and minor axes of the ellipse, $v_{1 c}$ and $v_{2 c}$ are the corrected signals, and $z_{x}$ and $z_{y}$ are the coordinates of the center of the ellipse $[12,13]$. The parameters $a, b, z_{x}$ and $z_{y}$ are obtained by fitting the waveform data $\left(V_{1}\right.$ and $\left.V_{2}\right)$ to an ellipse described by the equation $[12,13]$

$$
\left[\begin{array}{l}
x \\
y
\end{array}\right]=\mathbf{Q}(\alpha)\left[\begin{array}{ll}
a & 0 \\
0 & b
\end{array}\right]\left[\begin{array}{l}
\cos \theta \\
\sin \theta
\end{array}\right]+\left[\begin{array}{l}
z_{x} \\
z_{y}
\end{array}\right] .
$$

After substituting the parameters to fit the data to an ellipse, Eq. (5) is solved for [V1c;V2c] to obtain the corrected signals. The remaining steps for calculating detonation ve- 
locity from instantaneous phase are described in Vuppuluri et al. [1].

\section{Results and Discussion}

The validation of the experimental technique was done in Vuppuluri et al. using HMX [1]. Position-time plots resulting from applying the analysis process outlined in the previous section showed that the sample material detonation velocity was nearly steady from the initial position of the sample material, showing that the booster charge was well-matched to initiate the sample material to a steady velocity rapidly [1]. The authors measured the detonation velocity for $\mathrm{HMX}$ to be $7.15 \pm 0.04 \mathrm{~km} \mathrm{~s}^{-1}$ [1]. An uncertainty analysis presented in Vuppuluri et al., which showed that the uncertainty in detonation velocity was about 1.68 percent (about $120 \mathrm{~m} \mathrm{~s}^{-1}$ ) [1].

Detonation velocity measurements for CL-20 and CL-20/ $\mathrm{HP}$ were performed at powder densities of $1.327 \pm 0.052 \mathrm{~g} \mathrm{~cm}^{-3}$ and $1.311 \pm 0.084 \mathrm{~g} \mathrm{~cm}^{-3}$, respectively. Position-time plots of the CL-20/HP solvate and CL-20 are shown in Figure 3 and indicate that the detonation event is steady from the start. A linear curve fit was applied to each position-time curve and the slope resulting from this curve fit was taken to be the detonation velocity. The detonation velocity for the CL-20/HP solvate was found to be $7.21 \pm$ $0.34 \mathrm{~km} \mathrm{~s}^{-1}$. The detonation velocity for CL-20 was found to be $7.00 \pm 0.22 \mathrm{~km} \mathrm{~s}^{-1}$. The observed relative standard deviations were greater than the expected measurement uncertainty.

To determine enthalpy of formation, the effective molecular weight of the CL-20/HP solvate was calculated as $2 M_{\mathrm{CL}-20}+M_{\mathrm{HP}}$, yielding $910.38 \mathrm{~g} \mathrm{~mol}^{-1}$. Using Eqs. (1) through (3), the enthalpy of formation was found to be $748 \pm 128 \mathrm{~kJ} \mathrm{~mol}^{-1}$. The enthalpy of formation for $\varepsilon-\mathrm{CL}-20$ was measured to be $377 \mathrm{~kJ} \mathrm{~mol}^{-1}$ in Simpson et al. [14]. The enthalpy of formation for HP is $-187 \mathrm{~kJ} \mathrm{~mol}^{-1}$ [15]. Using these values, the enthalpy of formation for a physical mixture of CL-20 and HP in a 2:1 molar ratio is calculated at $567 \mathrm{~kJ} \mathrm{~mol}^{-1}$.

A new reactant for the $\mathrm{CL}-20 / \mathrm{HP}$ solvate was created in CHEETAH v7.0 with crystal density, molecular formula, and enthalpy of formation as the input [16]. Using a method previously described in Vuppuluri et al., relationships between detonation velocity and density for both CL-20 and CL-20/HP were found to be of the form:

$U=a \rho_{0}+b$,

where $\rho_{0}$ is powder density, $U$ is detonation velocity, and $a$ and $b$ are fitting parameters. The measured detonation velocities were corrected to a powder density of $1.4 \mathrm{~g} \mathrm{~cm}^{-3}$ using the equation

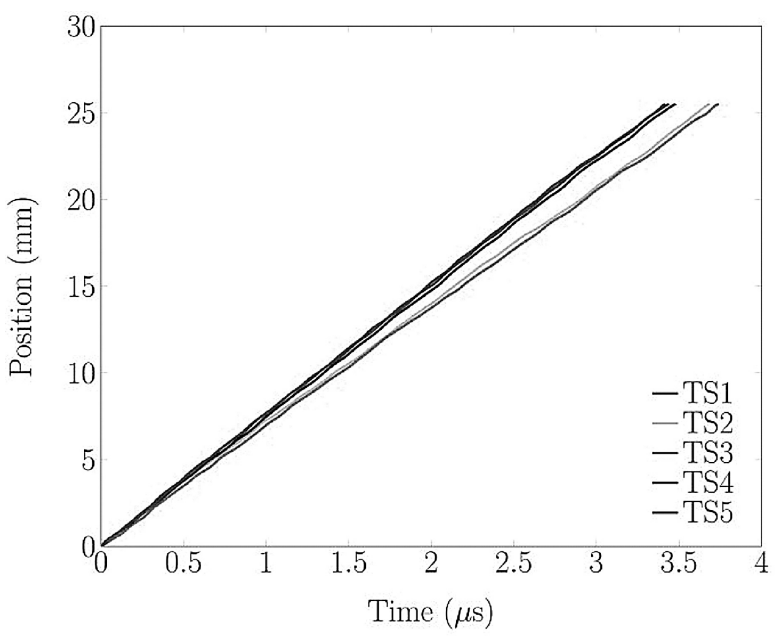

(a)

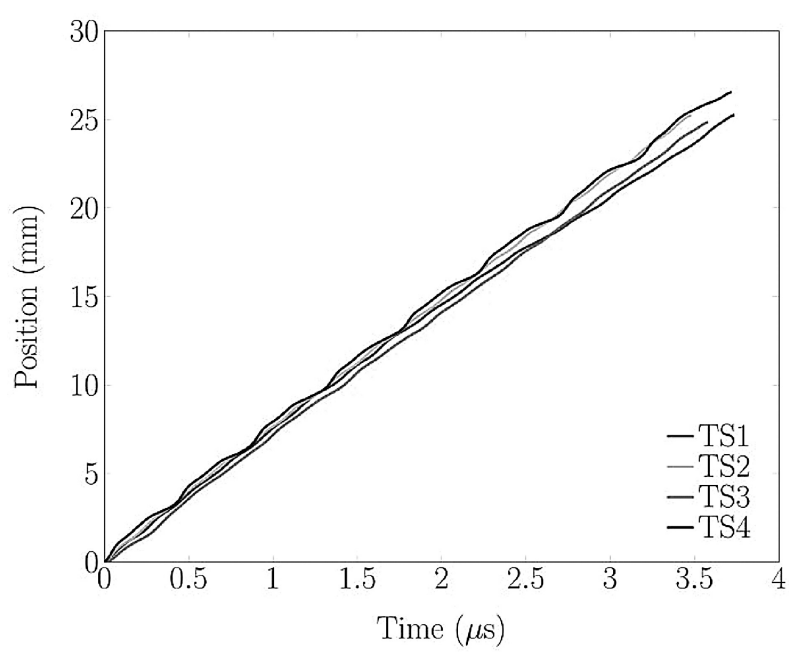

(b)

Figure 3. (a) Position vs. time plots for CL-20/HP solvate detonation tests. (b) Position vs. time plots for CL-20 detonation tests.

$U_{\text {corr }}=U_{\text {unc }}+a\left(1.4-\rho_{0}\right)$

where $U_{\text {corr }}$ and $U_{\text {unc }}$ are respectively the corrected and uncorrected velocities, and $\rho_{0}$ is the charge density. The parameter $a$ is the value found by applying the curve fit given in Eq. (7) to calculated velocities found from CHEETAH. It was assumed that the density-detonation velocity relationship for measured detonation velocity would be the same as that for the predicted detonation velocity.

The measured detonation velocity for CL-20/HP corrected to a charge density of $1.4 \mathrm{~g} \mathrm{~cm}^{-3}$ was found to be $7.55 \pm 0.092 \mathrm{~km} \mathrm{~s}^{-1}$. The measured detonation velocity for CL-20 corrected to charge density of $1.4 \mathrm{~g} \mathrm{~cm}^{-3}$ was found to be $7.21 \pm 0.064 \mathrm{~km} \mathrm{~s}^{-1}$. The standard deviations for both CL-20 and CL-20/HP after density correction were lower than what was seen with the uncorrected velocities, indicat- 
ing that the large variances were due to variations in charge density.

The measured detonation velocities for $\mathrm{HMX}, \mathrm{CL}-20$, and $\mathrm{CL}-20 / \mathrm{HP}$ at a powder density of $1.4 \mathrm{gcm}-3$ along with $\mathrm{CHEETAH}$ predictions at the same density are shown in Figure 4. The error bar on the predicted detonation velocity for the solvate was obtained by calculating detonation velocity in $\mathrm{CHEETAH}$ with enthalpy of formation between $\left[\Delta \overline{H_{f}^{\circ}}-\sigma, \Delta \overline{H_{f}^{\circ}}+\sigma\right]$, where $\Delta \bar{H}_{f}^{\circ} \bigcirc$ is the average measured enthalpy of formation and $\sigma$ is the standard deviation. The measured detonation velocity for $\mathrm{CL}-20 / \mathrm{HP}$ at $1.4 \mathrm{~g} \mathrm{~cm}^{-3}$ was more than a standard deviation above the predicted detonation velocity, which one would ordinarily not expect

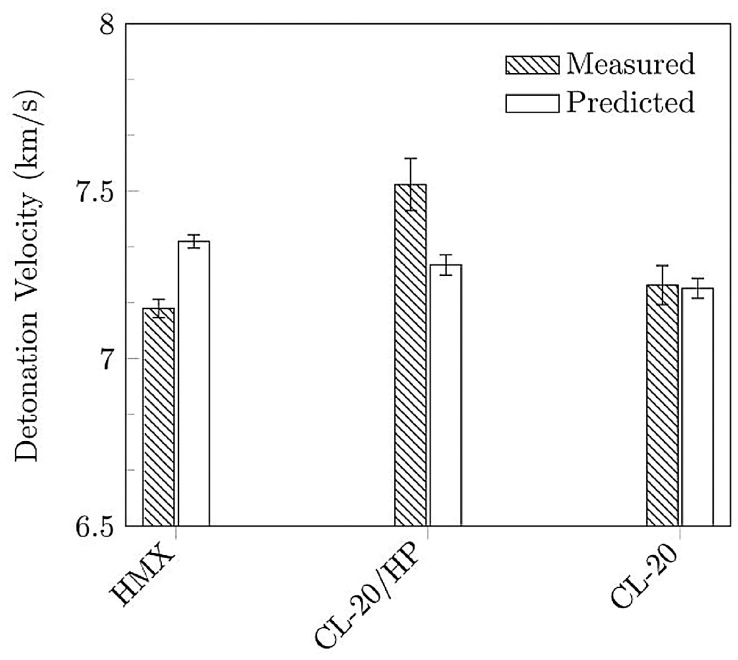

(a)

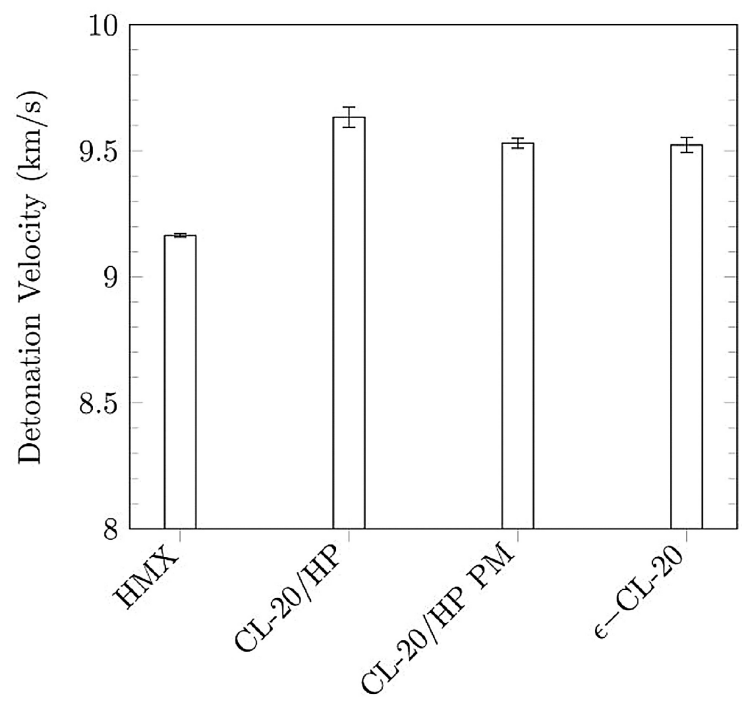

(b)

Figure 4. (a) Predicted vs. measured detonation velocities at $1.4 \mathrm{~g} \mathrm{~cm}^{-3}$. (b) Predicted detonation velocities at TMD. given that the assumptions used in CHEETAH are valid in the limit of infinite charge diameter. We also note that the predictions in CHEETAH at this density did not agree with the observed results. The measured detonation velocity of the solvate at a loading density of $1.4 \mathrm{~g} \mathrm{~cm}^{-3}$ was $300 \mathrm{~m} \mathrm{~s}^{-1}$ faster than that of $\mathrm{CL}-20$ at the same loading density, which was larger than the CHEETAH prediction of only $70 \mathrm{~m} \mathrm{~s}^{-1}$. CHEETAH calculates the detonation velocity from the expected product composition at the $C-J$ state using a desired EOS, which was the JCZ3 EOS for this work. A deviation between measured and predicted velocities for a particular test explosive may indicate that the EOS is improperly calibrated for the test explosive. Furthermore, the EOS could also fail to predict the $C-J$ state accurately at the high porosities found in this work (about 30 percent). Predicted detonation velocities of $\mathrm{HMX}, \mathrm{CL}-20, \mathrm{CL}-20 / \mathrm{HP}$ solvate, and $\mathrm{CL}-20 / \mathrm{HP}$ physical mix at their respective TMDs are shown in Figure 4. From Figure 4, it can be seen that the detonation velocity of the CL-20/HP solvate is predicted to be about $100 \mathrm{~m} \mathrm{~s}^{-1}$ faster than that of CL-20, despite being less dense than $\mathrm{CL}-20$. The predicted detonation velocity of the CL-20'HP solvate at TMD also exceeds that of a formulation or physical mixture of the components. The calculated TMD of the physical mixture is $2.013 \mathrm{~g} \mathrm{~cm}^{-3}$, which is less dense than the solvate, resulting in a lower detonation velocity. Furthermore, of all the energetic cocrystals or solvates of $\mathrm{CL}-20$ reported previously, the $\mathrm{CL}-20 / \mathrm{HP}$ solvate is the only example of a CL-20 cocrystal where the TMD of the formulation is less dense than the TMD of the cocrystal or solvate.

\section{Conclusion}

In this work, a comparison for detonation velocity of a HP solvate of CL-20 and neat CL-20 is presented. The detonation velocity of the solvate at an overall density of $1.4 \mathrm{~g} \mathrm{~cm}^{-3}$ was found to be $300 \mathrm{~m} \mathrm{~s}^{-1}$ faster than that of the $\mathrm{CL}-20$ at the same density. The enthalpy of formation was also measured, which was used to obtain thermochemical predictions with $\mathrm{CHEETAH}$. CHEETAH predicted that the solvate would detonate only $70 \mathrm{~ms}^{-1}$ faster than $\mathrm{CL}-20$ at a powder density of $1.4 \mathrm{~g} \mathrm{~cm}^{-3}$, which was lower than what was measured.

It was predicted in CHEETAH that the solvate would detonate $100 \mathrm{~m} \mathrm{~s}^{-1}$ faster than $\varepsilon-\mathrm{CL}-20$. This shows the potential benefit of solvate formation in obtaining enhanced detonation performance. While cocrystals of CL-20 (HMX/CL-20 and MDNT/CL-20) have been reported as having high densities, none of these are as dense as CL-20. Consequently, thermochemical calculations do not predict that any of these cocrystals will detonate faster than $\varepsilon-C L-20$. We further note that although the orthorhombic CL-20/HP solvate has a lower density than that of $\varepsilon-C L-20$, the solvate has a superior oxygen balance. Despite having a lower TMD than that of $\varepsilon-C L-20$, the solvate is predicted to detonate faster 
than CL-20, making it the only energetic CL-20 cocrystal or solvate to exhibit this effect.

In addition, as mentioned previously, every cocrystal or solvate of CL-20 with the exception of the HP solvate analyzed in this work has a lower density than that of CL-20, which is likely to undermine the performance of these materials relative to $\mathrm{CL}-20$.

\section{Acknowledgements}

This material is based upon work supported by the U. S. Army Research Laboratory and the U. S. Army Research Office under contract/grant number W911NF-13-1-0387.

\section{References}

[1] V. S. Vuppuluri, P. J. Samuels, K. C. Caflin, I.E. Gunduz, S. F. Son, Detonation Performance Characterization of a Novel CL-20 Cocrystal Using Microwave Interferometry. Propellants Explos. Pyrotech. 2018, 43, 38-47.

[2] K. A. Kersten, R. Kaur, A. J. Matzger, Survey and analysis of crystal polymorphism in organic structures. Int. Union Crystallogr. J. 2018, 5, 124-129.

[3] A. T. Nielsen, A. P. Chafin, S. L. Christian, D. W. Moore, M. P. Nadler, R. A. Nissan, D. J. Vanderah, R. D. Gilardi, C. F. George, J. L. Flippen-Anderson, Synthesis of polyazapolycyclic caged polynitramines. Tetrahedron 1998, 54, 11793-11812.

[4] K. B. Landenberger, A. J. Matzger, Cocrystals of 1,3,5,7-Tetranitro-1,3,5,7-tetrazacyclooctane (HMX). Cryst. Growth Des. 2012, $12,3603-3609$.

[5] J. C. Bennion, N. Chowdhury, J. W. Kampf, A. J. Matzger, Hydrogen Peroxide Solvates of 2,4,6,8,10,12-Hexanitro-2,4,6,8,10,12hexaazaisowurtzitane. Angew. Chem. Int. Ed. 2016, 55, 1311813121; Angew. Chem. 2016, 128, 13312-13315.

[6] O. Bolton, A. J. Matzger, Improved stability and smart-material functionality realized in an energetic cocrystal. Angew. Chem.
Int. Ed. 2011, 50, 8960-8963; Angew. Chem. 2011, 123, 91229125.

[7] O. Bolton, L. R. Simke, P. F. Pagoria, A. J. Matzger, High Power Explosive with Good Sensitivity: A 2:1 Cocrystal of CL-20:HMX. Cryst. Growth Des. 2012, 12, 4311-4314.

[8] S. R. Anderson, P. Dubé, M. Krawiec, J. S. Salan, D. J. am Ende, P. Samuels, Promising CL-20- based energetic material by cocrystallization. Propellants Explos. Pyrotech. 2016, 41, 783-786.

[9] G. F. Cawsey, J. L. Farrands, S. Thomas, Observations of Detonation in Solid Explosives by Microwave Interferometry. Proc. $R$. Soc. A: Math. Phys. Eng. Sci. 1958, 248, 499-521.

[10] P. L. M. Heydemann, Determination and correction of quadrature fringe measurement errors in interferometers. Appl. Opt. 1981, 20, 3382-3384.

[11] E. Layer, K. Tomczyk, Hilbert Transform. in: Signal Transforms in Dynamic Measurements, Springer, 2015, p. 107.

[12] W. Gander, G. H. Golub, R. Strebel, Least-squares fitting of circles and ellipses. BIT 1994, 34, 558-578.

[13] D. E. Kittell, J. O. Mares, S. F. Son,Using time-frequency analysis to determine time-resolved detonation velocity with microwave interferometry. Rev. Sci. Instrum. 2015, 86, 044705.

[14] R. L. Simpson, P. A. Urtiew, D. L. Ornellas, G. L. Moody, K. J. Scribner, D. M. Hoffman, CL- 20 performance exceeds that of HMX and its sensitivity is moderate. Propellants Explos. Pyrotech. 1997, 22, 249-255.

[15] B. Ruscic, R. E. Pinzon, M. L. Morton, G. von Laszevski, S. J. Bittner, S. G. Nijsure, K. A. Amin, M. Minko, A. F. Wagner, Introduction to active thermochemical tables: several key enthalpies of formation revisited. J. Phys. Chem. A, 2004, 108, 9979-9997.

[16] S. L. Bastea, L. E. Fried, K. R. Glaeseman, W. M. Howard, I. F. W. Kuo, P. C. Souers, P. A. Vitello, Cheetah 7.0 thermochemical code. Lawrence Livermore National Laboratory, Livermore, CA, 2012.

Manuscript received: June 25, 2018 Revised manuscript received: October 14, 2018 Version of record online: January 17, 2019 